SLAC-PUB-11490

Sept 2005

\title{
A Novel Technique for the Production of Large Area Z-coordinate Readout Planes for the BABAR Muon System *
}

\author{
M. R. Convery ${ }^{\mathrm{a}}$, P. C. Kim ${ }^{\mathrm{a}}$, H. P. Paar ${ }^{\mathrm{b}}$, C. H. Rogers ${ }^{\mathrm{a}}$, \\ R. H. Schindler ${ }^{\text {a }}$, S. K. Swain ${ }^{\mathrm{a}, *}$, C. C. Young ${ }^{\mathrm{a}}$ \\ ${ }^{a}$ Stanford Linear Accelerator Center, Stanford, CA 94309, USA. \\ ${ }^{\mathrm{b}}$ Department of Physics, University of California, San Diego, CA 92093, USA.
}

\begin{abstract}
The BABAR detector, at the Stanford Linear Accelerator Center is a general purpose detector for the study of $e^{+} e^{-}$interactions at the $\Upsilon(4 S)$ resonance. BABAR's muon detection system consists of two parts: a hexagonal barrel region and two planar endcap regions each containing 18 layers of iron $(\sim 3.6 \lambda)$, with resistive plate chambers within the inter-iron gaps. These chambers have suffered deterioration in performance over the past few years and are being replaced by limited streamer tube chambers in the barrel. Each layer of the system consists of a set of up to 10 streamer tube modules oriented parallel to the beamline providing the azimuthal coordinate $(\Phi)$ and a single "Z-plane" with strips oriented perpendicular the streamer tubes providing the coordinate $(\mathrm{Z})$ along the beamline. The large area Z-planes (up to $12 \mathrm{~m}^{2}$ ) are $1 \mathrm{~mm}$ thick and contain 96 strips that detect the induced charge from avalanches on the streamer tube wires. This paper reports on the novel construction technique of the Z-planes.
\end{abstract}

Key words: Muons, Limited Streamer Tube, BABAR Experiment PACS: 95.85.Ry, 99.55.Vj

^ Work supported by Department of Energy contract DE-AC02-76SF00515.

* Corresponding author.

Email address: sanjay@slac.standford.edu (C. C. Young). 


\section{INTRODUCTION}

The primary goal of the $B_{A} B A R$ experiment is to study $\mathrm{CP}$ violation in $\mathrm{B}$ meson decays. The detector used for this experiment consists of several sub-systems for the tracking and particle identification [1]. The instrumented flux return (IFR) is used for muon and neutral hadron identification by placing active detectors between the 18 layers of the flux return iron. The existing system utilizes resistive plate chambers (RPC) [2] in the gaps of iron. These chambers are now being replaced with plastic limited streamer tubes (LST) [3]. In

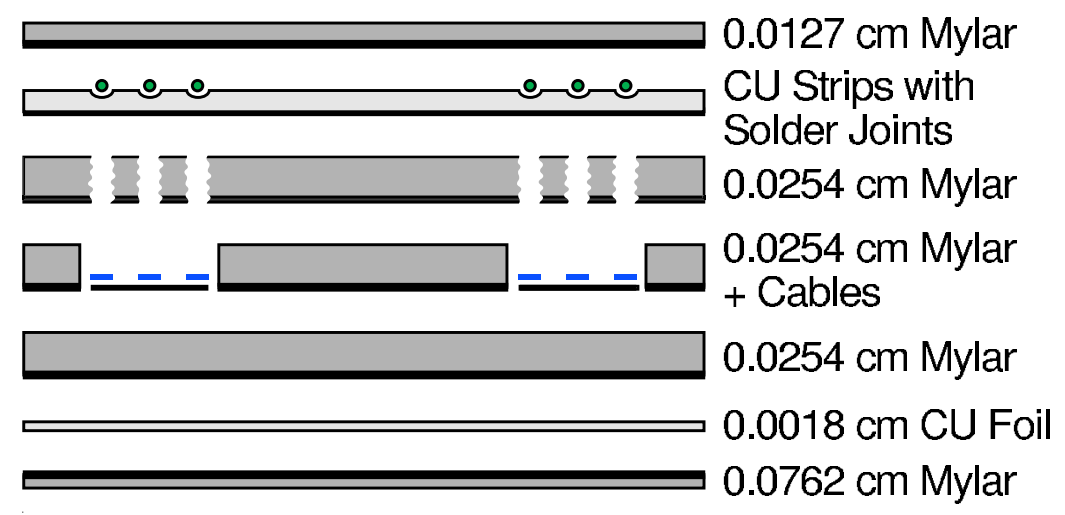

Fig. 1. Layers of different materials used for a Z-plane. The thick darkline in each layer is the adhesive side. The dashed lines on the fourth layer from the bottom are the cable locations. The dots in the second layer from the top are the solder joints. The cables are soldered to the strips through the pre-punched holes in layer between them (shown as a narrow cut out on the layer).

the past, these ionization detectors have been used in the ZEUS [4], LEP [5], SLD [6] and PHENIX [7] experiments and have shown satisfactory performance with high efficiency and reliability once tested and adequately burned in. In the $B_{A} B_{A R}$ LST system, the ionization charge is collected by the wires in each tube, through a high voltage blocking capacitor to provide the $\Phi$ coordinate directly. Low impedance cathode strips positioned outside outside the LST module and running orthogonal to the wires are capacitively coupled to nearby wires, producing an induced charge signal giving the $\mathrm{Z}$ coordinate. The charge is shared across 3 or 4 strips and is centroided to yield a $\mathrm{Z}$ resolution of about $2 \mathrm{~cm}$. These cathode or Z-planes, are built for each layer in a single large plane, using a novel thermal-vacuum laminating process. This flexible technique allowed us to reliably produce 72 Z-readout planes up to $3 \times 4 \mathrm{~m}^{2}$ and $1 \mathrm{~mm}$ thick. The vacuum technique is well matched to achieve $\pm 25 \mu \mathrm{m}$ thickness tolerance across the large area and gave us flexibility to produce 12 different versions of the plane, with the same tooling. This paper details the design and construction technique for manufacturing the Z-planes. 


\section{MATERIALS, DIMENSIONS AND CONSTRUCTION OVERVIEW}

The $B_{A} B_{A R}$ IFR barrel region is built up of sextants currently containing 18 layers each of RPC chambers filling the $26 \mathrm{~mm}$ wide gaps of the flux-return iron. In the upgrade, 12 of the 18 gaps are filled with new LST chambers and six of the gaps are filled with brass absorber, to improve muon-hadron separation. Each of the 12 replacement layers in a sextant requires a single large Z-plane to be installed in the gap before the narrow LST modules are inserted. Each of the 12 Z-plane layers are unique, both dimensionally, and in strip and signal routing. The Z-planes vary in size to match the different slot widths in the iron structure which vary from $1908 \mathrm{~mm}$ for the innermost layer, to $3110 \mathrm{~mm}$ for the outermost layer. The length of each Z-plane is $3719 \mathrm{~mm}$, except for the outermost one which is $370 \mathrm{~mm}$ shorter due to installation interferences. The thickness of each Z-plane is precisely controlled to be $1.00 \pm 0.02 \mathrm{~mm}$ to guarantee clearance in the gaps for the module insertion. To control this

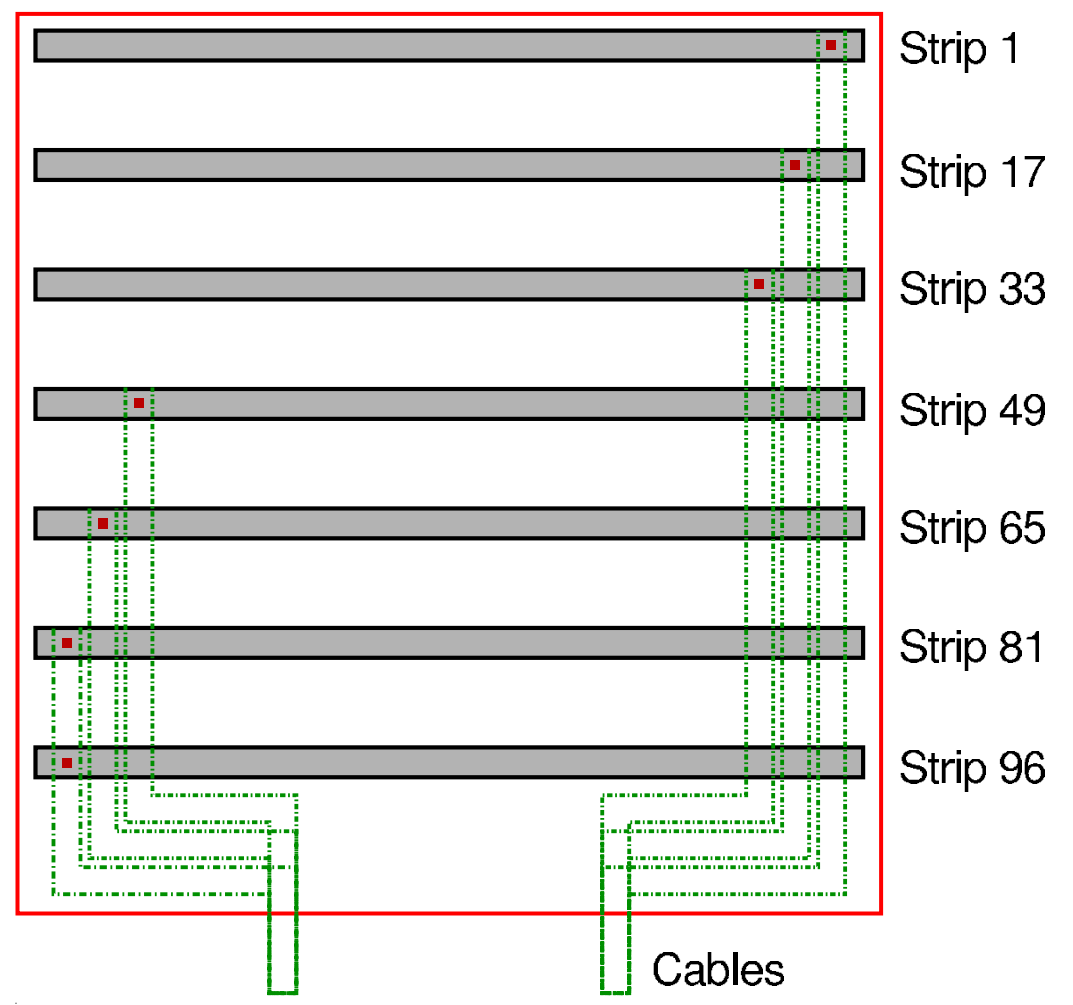

Fig. 2. Top view of a Z-plane. The shaded rectangles are copper strips. The dashed green lines are the cables. There are 96-strips in each plane although only seven strips are shown as an illustration.

thickness, a vacuum lamination technique was adopted and suitable materials were chosen. The Z-plane is built up out of layers of Mylar, copper foils, and Mylar ribbon cables, which are then vacuum laminated together in a single operation, using a combination of acrylic contact adhesives and a dry-thermal setting adhesive. The thickness of each individual layer of material is tightly 
controlled, including the adhesives. Using a vacuum system rather than a roll process guarantees uniform pressure over the entire surface during the thermal adhesive setting process. Heating of the materials is applied uniformly and simultaneously over the entire surface of the plane. Under these conditions, the adhesives undergo very little flow, resulting in an extremely repeatable process that yields precise control of the total thickness.

The schematic of the layout of one such Z-plane is shown in Fig. 1 and Fig. 2. Each plane is composed of a common $\mathrm{Cu}$-foil ground plane, separated with a thick dielectric film, from a layer of 96 parallel conductive readout strips (each $35 \mathrm{~mm}$ wide and separated by $2 \mathrm{~mm}$ ) running the width of the plane. The strips are soldered to embedded readout cables which form a part of the plane itself. These cables come out to one end of the detector plane, where the readout electronics are located.

The large ground plane utilizes $18 \mu \mathrm{m}$ thick copper foil produced by Gould Electronics Inc. [8] and delivered precut in widths up to 1.06 meters. While it was possible to get wider rolls, it would have been impractical to handle this very thin material if it had been any wider. The foil itself is electro-deposited rather than rolled and arrives in a soft annealed state. It is shiny on one side and has been passivated on the other side for better adhesion to the acrylic adhesives. For dielectrics, each Z-plane uses one layer each of $0.762 \mathrm{~mm}$ (3 mil)

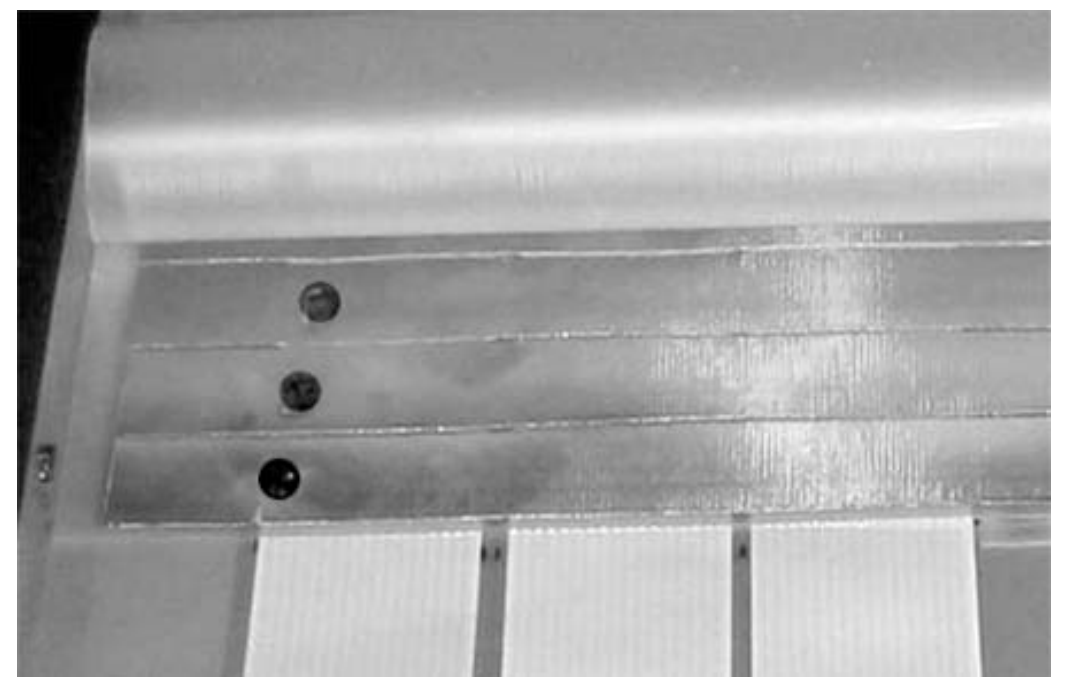

Fig. 3. The Z-strips soldered to 16-channel cables before lamination.

and $0.127 \mathrm{~mm}$ ( 5 mil) thick and three layers of $0.254 \mathrm{~mm}(10 \mathrm{mil})$ thick PET film (similar to trade name, Mylar). For convenience we will use mil, which is equal to $0.0254 \mathrm{~mm}$, while describing the thickness of the materials. The 3 mil and 5 mil films are prepared by the manufacturer (MACTAC) [9] with 2 mils each of a dry thermal setting adhesive on one side. The 10 mil film has 3 mils of adhesive and 7 mils of Mylar. These adhesives set in about 5 minutes at $\sim 90^{\circ} \mathrm{C}$. The film is delivered in rolls of standard widths $(30.5,83.8$ and 
$96.5 \mathrm{~cm}$ ), and lengths of 76.2 to 152.4 meters. Because the Z-planes are of 12 different widths, a local company was contracted to precisely cut and rewind these rolls to the distinct widths which could be used to build up the full desired width of any layer using up to 5 pieces. For structural integrity, we arranged the Mylar widths and their sequencing in such a manner that any gaps between pieces of Mylar (and/or foils) in any layer would never line up closer than $\sim 6 \mathrm{~cm}$. In addition, a $2 \mathrm{~mm}$ gap between pieces was always left as we found that it improved the adhesion of layers and added to the strength of the joints.

The copper strips themselves are made from 1.5 mil thick copper foil tape, custom manufactured by Universal Tape Co [10] cut to $35 \mathrm{~mm}$ width and backed with an acrylic contact adhesive of thickness $\sim 0.5$ mils. The 33 meter rolls came with a peal-off paper backing suitable for use in a commercial tape laying machine. The last unique component of the design is the use of custom

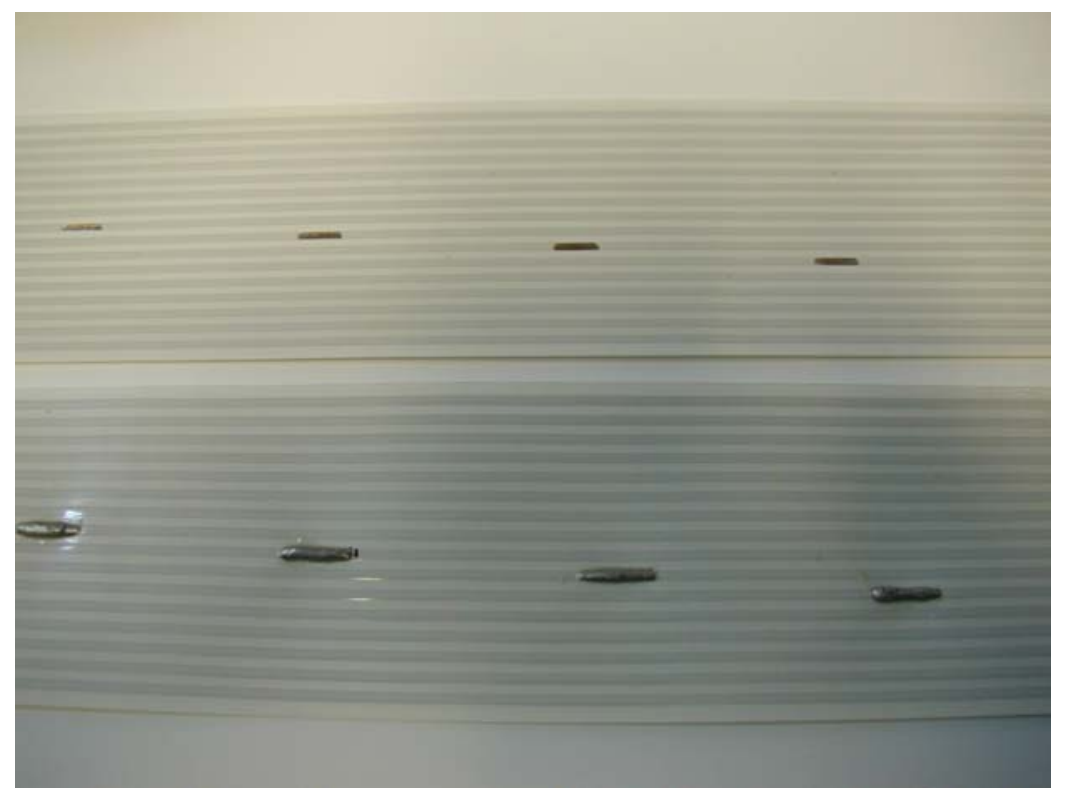

Fig. 4. 16-channel cable. The gap between the two pre-tinned channels matches the center of the adjacent strips.

fabricated flat flexible cables by PARLEX Corp [11] to carry signals from the strips to the electronics. These 16 conductor cables are made by roll lamination of Mylar/adhesive and pre-tinned flat copper conductors. They are 3 mils thick having 100 mil wide conductors on 100 mil spacing. These cables have similar material specifications as the rest of the Z-plane lamination components, and thus allowed us to incorporate them directly into the Z-plane thermal vacuum lamination processing. PARLEX prepared the cable with a repeating step pattern of 16 laser stripped vias $(8 \mathrm{~mm}$ long $\times 2.54 \mathrm{~mm})$ on one side of the cable to allow the final soldering to the copper strips as shown in Fig. 3. The cables were terminated with the sockets [12] that were crimped onto the cables by a local company [13] before the plane asssemby. 


\section{DETAILED ASSEMBLY OF A PLANE}

The basic assembly process proceeds as follows. All components of the plane have been precut to length and width, and inspected for proper dimension. A layer of 3 mil thick Mylar film, adhesive-side up, is affixed to the polished metal top of a vacuum lamination table with plating tape (suitable for high temperatures) along the edges. The film is not sufficiently wide so several pieces are used, and allowed to overlap each other about $5 \mathrm{~mm}$ along the length to guarantee a continuous film on this outer layer. The lapping of this joint creates a 1 mil high ridge.

The Mylar is followed by the ground-plane layer of cu-foil, with the passivated side down towards the adhesive. Again, the width is built up out of two or three pieces of foil, overlapped about $5 \mathrm{~mm}$ and soldered together at several points along the edge. The foil is pre-dimensioned to be $5 \mathrm{~mm}$ narrower than the underlying Mylar, so it will be fully contained. Next, a layer of 10 mil film is placed adhesive-side down onto the foil. Throughout the balance of the layout, the Mylar film is dimensioned to extend $5 \mathrm{~mm}$ beyond the copper plane. Thus, these first two layers of film fully encapsulate, electrically insulate, and protect the delicate copper foil. All following layers of film are placed with the adhesive

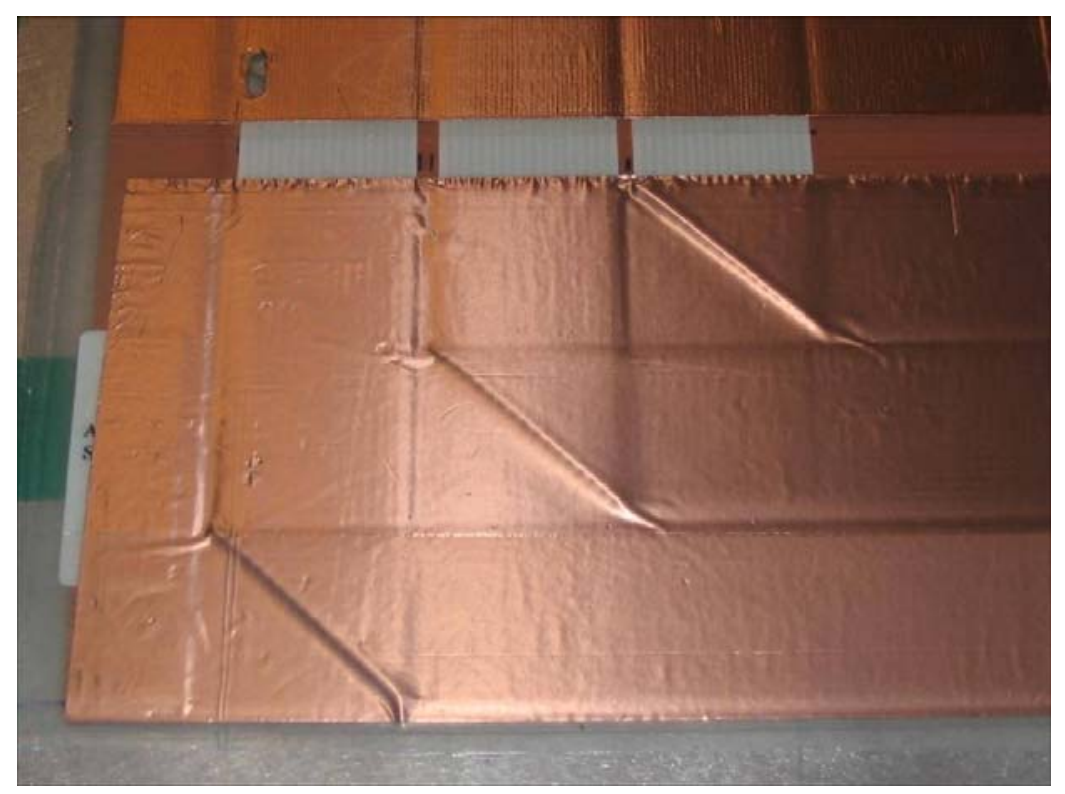

Fig. 5. The cable is folded around the corner of the plane. The diagonal creases are the folded positions.

side down. A second layer of 10 mil film is laid down. This layer is modified to incorporate the flat Mylar cables that will carry the signals out of the Zplane. Sections of the film are cut away to form channels where the cables lie, to prevent adding thickness. This results in several non-contiguous pieces of film which could shift position if not secured in some way. To avoid the problem, 
the film is carefully positioned and then tacked down intermittently along one edge with a hot iron. These tacks are free to expand in one direction without buckling and remelt during the final lamination. The cables themselves are secured to the 10 mil layer below by using transfer tape of similar width, with 1 mil acrylic adhesive. The tape is peeled on one side and applied to the backside of the cable in advance. The other side is peeled to expose the adhesive, only as it is laid down onto the Mylar in its desired location. To insure encapsulation of the cables, and the individual mylar pieces by the subsequent 10 mil layer, we found that it was necessary to leave $2 \mathrm{~mm}$ margins around the cable, and between non-contiguous mylar pieces, just as was done in building up the total width.

The mylar cable is positioned so that the pre-stripped vias will ultimately align with the copper tape strips. The exposed conductors are pre-tinned with low temperature solder, to leave prominent ridges as shown in Fig. 4. The next layer of $10 \mathrm{mil}$ film has $30 \mathrm{~cm}$ wide edge regions that have been pre-punched using a rule die with a series of large slots $(12 \mathrm{~mm}$ by $5 \mathrm{~mm})$, arranged to overlay the vias in the cables. The 96 strips of cu tape are applied across the width of this layer using a tape laying machine, described later. Each cu strip crosses only one of the punched slots, and hence one of the exposed, pre-tinned conductors. The copper tape is pressed firmly into the slot, onto the pre-tinned cable below, to leave a distinct impression of the conductor. A sharp knife is used to make a slice through the cu tape along the conductor. The tape is stretched further, exposing the tinning. A soldering iron is used to heat and flow the solder onto the top surface of the tape, while adding more solder to the joint. Because of the large slot and the 10 mil thick material, the solder can be 5 mils thick without creating a ridge in the plane. This completes the electrical connection to the cable as shown in Fig. 3. There are six 16-conductor cables to collect the signals from all 96 strips. Three such cables come out on each side of the plane. These cables are folded around the corner of the plane once as shown in Fig. 5, and then brought back towards the center, where they are folded again. The last fold places the 3 cables on one side on top of each other, separated by $85 \mathrm{~cm}$ distance from the set of three, on the other side. The $16 \mathrm{~cm}$ wide region where the folding occurs, has the 10 mil Mylar removed, so as not to add thickness. For shielding, an $18 \mathrm{~cm}$ wide strip of copper tape covers the area, and is soldered to the ground plane. Fig. 3, Fig. 5 and Fig. 8 show the cables before and after folding, respectively, and emerging from the plane.

The application of the 96 copper tape strips was accomplished with a large precision tape laying fixture shown in Fig. 6. The tape laying machine and the support structure were built to maintain the $2 \pm 0.5 \mathrm{~mm}$ gap, between the strips over $3.2 \mathrm{~m}$ in length. The fixture consisted of a heavy rigid box beam that spanned the assembly and laminating table $(\sim 4.5 \mathrm{~m})$ and traveled back and forth on Thomson rails with nearly zero-lash re-circulating-ball type roller assemblies. This provided a very smooth, non-binding carrier for a commercial 


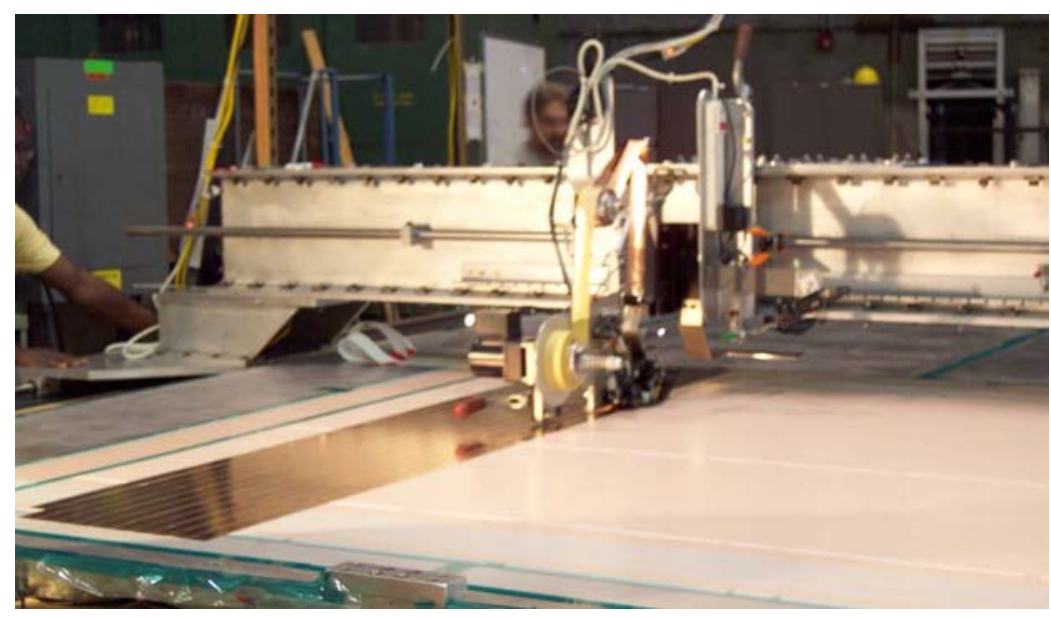

Fig. 6. Taping machine and carrier used for laying down the strips.

automatic tape dispenser rented from Straub Design Company [14]. The box beam and rail assembly was pre-aligned to the laminating table top and had a slotted guide that indexed the tape-dispenser at the 96 desired positions along the box-beam, where the copper tape strips were to be laid down.

The tape dispenser was primarily driven by the action of tape being pulled from it. An electric motor actively wound up the tape backing as it was peeled from the copper foil. There was also a solenoid-operated guide-roller that aligned and pressed the tape against the Mylar on the table surface, and a knife blade to cut it off cleanly at the end of an application cycle. These actions were controlled by one operator watching the tape head on a video monitor, while a second operator alternately indexed the dispenser to its next position for application of the next tape. The time required to lay down 96 strips was initially dominated by the time to re-thread new tape onto the tape laying head. We subsequently modified the machine to handle triple length $(\sim 92 \mathrm{~m})$ rolls of tape, which we pre-spliced in advance, in a separate setup. A typical plane required $\sim 2$ hrs time to lay down all 96 strips.

All the electrical connections of the ground planes, strips and cables are tested, before adding a final protective layer of 5 mil film. We measured the capacitance and resistance between the copper ground plane and the strips and the capacitance and resistance of the strips through the cables. This guarantees good solder joints, as well as checking for unexpected connections between the strips, which are geometrically close together.

\section{VACUUM LAMINATION}

The assembly procedure described thus far was carried out on a large, $1.27 \mathrm{~cm}$ thick table top made from three sections of aluminum plate. The top surface 
measured $4 \times 4$ meters. The aluminum plates were butt joined by welding a series of mating blocks along each mating edge of what would become the underside of the table. The blocks contained 3 steel bushings and 3 bolts which were used to draw the edges together tightly. Conventional glass-insulated heating tapes were clipped to the underside of the aluminum, and were then covered with high temperature aluminum tape to insure contact. These ran across the width at $\sim 30 \mathrm{~cm}$ intervals and provided about $15 \mathrm{KW}$ of heating capacity. They were independently controlled by commercial rheostats. A set of 28 thermocouples were also installed into blind holes, on the underside between the tapes, to form a uniform measurement grid.

A support table was fabricated from flame retardant plywood covered with $12 \mathrm{~cm}$ of fiberglass insulation. A periodic array of threaded rod levelers was installed from the underside, and stuck up through the battens of insulation. The fully instrumented aluminum plate was next flipped over, and allowed to rest on top of the thick fiberglass insulation pad. The levelers were adjusted from underneath to uniformly support the aluminum plate using a laser level for reference. The plate was then chemically cleaned and mechanically polished and buffed to remove large scratches. Covering the table with additional insulation, a little experimentation yielded a suitable sequence of setpoints for the heater tapes, which could reproducibly could bring the table surface to $\sim 90 \pm 5^{\circ} \mathrm{C}$ in less than an hour.

With the Z-plane layers stacked up on the aluminum table surface the process of thermal and vacuum lamination begins. A thick bleeder cloth was first laid down over the entire Z-plane assembly. This was followed by sheets of commercial nylon vacuum-bagging material. The material could be assembled in pieces using PET film tape to join them along their seams. The vacuum bag was extended about $10 \mathrm{~cm}$ beyond the perimeter of the plane. The purpose of the bleeder cloth was to provide an "air space" between the laminate and the vacuum bag, to provide a pumping path for the air to be removed evenly from the entire surface. This prevented the formation of trapped bubbles under the vacuum bag. To provide vacuum, a long poly-flow tube was perforated and clipped to the aluminum plate under the vacuum bag and just outside of the assembled Z-plane on both long sides. One end of the tube was attached to a pump-out hole on the aluminum plate surface. The vacuum pump pulled air downward through this hole. This "C" shaped spacer and some additional bleeder cloth helped maintain an unobstructed path from the laminate to the pumpout hole, through the table. The vacuum bag sheets were sealed down to the aluminum table all along their perimeter by $5 \mathrm{~cm}$ wide PET film tape, custom made with a high-temperature adhesive. Leaks were generally not a problem and could be found visually and repaired. Once the laminate was pumped down successfully to $10^{-3}$ Torr, fiberglass insulation battens were distributed over the entire surface, and the heat cycle was initiated. The table was left at the operating point of about $90^{\circ} \mathrm{C}$ for about 5 minutes and then 


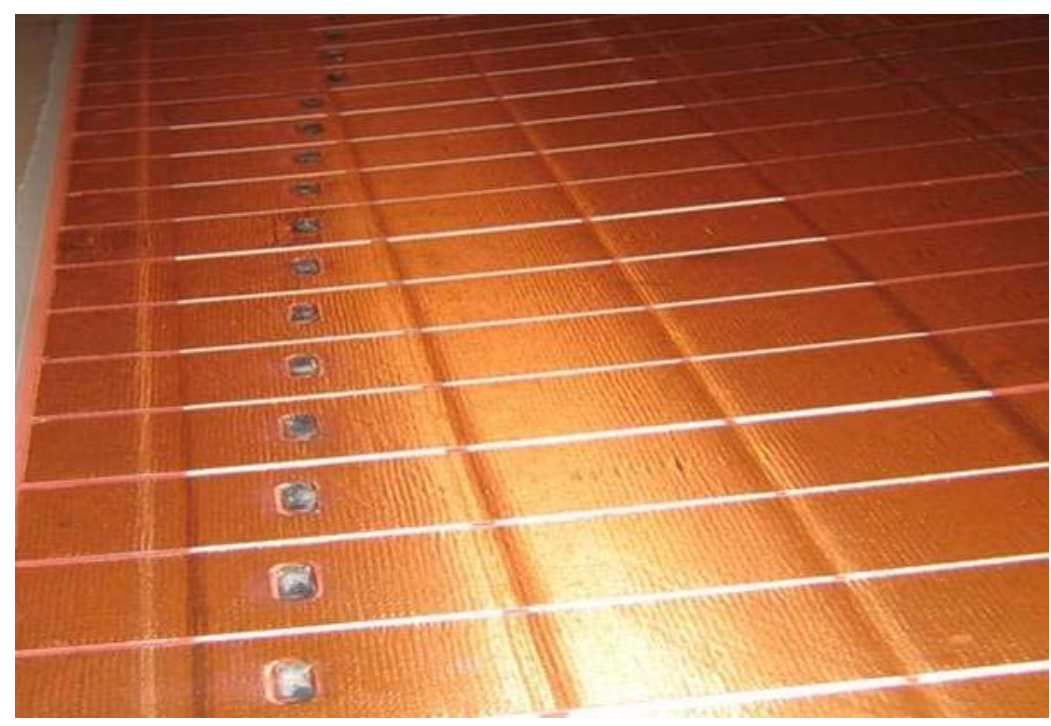

Fig. 7. The strips soldered to 16-channel cables after lamination. The three distinct white bands are the cables in the plane.

the heaters were all shut off. The cool down could be expedited considerably by removing the insulation blankets and positioning ventilation fans, once the temperature was well below the adhesive's melting point. A warm-up and cool-down cycle usually required two to three hours. Fig. 7 and Fig. 8 show the plane after lamination.

\section{FINAL PROCESSING AND PRODUCTION EXPERIENCE}

After each Z-plane was laminated, a visual inspection of the lamination quality was followed by a final resistance and capacitance check of each strip. The observed and expected values for each strip were compared. The capacitances range from $3.9 \mathrm{nf}$ to $5.7 \mathrm{nf}$ and are divided between the strip and the cable itself. The Z-planes were then transferred to a finishing table. During handling of the Z-planes, we kept them flat and well supported. On the finishing table, the Mylar edges were trimmed to final width and length. The signal cables were also trimmed to length, and terminated with pins and connectors. A final check of the continuity was performed.

During the initial phase of the production, the solder joints between the embedded cable and the strips were found to be susceptible to occasional breakage $(0.7 \%)$. It appeared that flexing of the large plane during handling had the potential to break the early solder joints. To improve reliability, we increased the area of each solder joint by stripping more of the cable insulation in length and width, by widening the cut in the cu-strip, by mechanically cleaning the conductor just prior to soldering, and by adding more solder to the joint. In- 


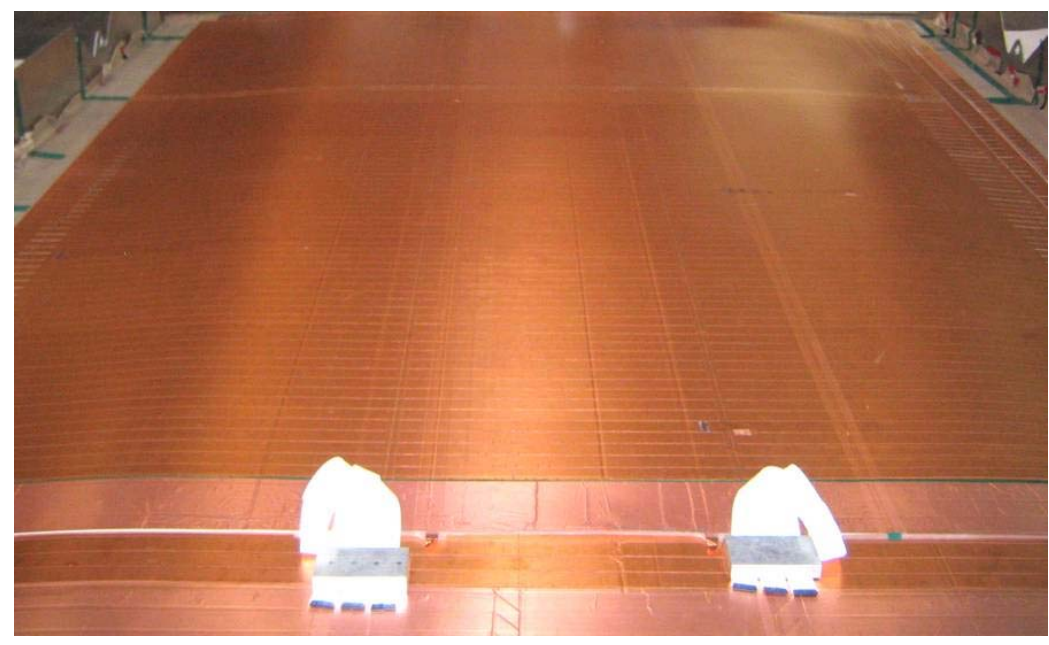

Fig. 8. View of a Z-plane after completion. The white ribbons shown in picture are the cables coming out of the plane.

creasing the area of the joint increased it strength. The cleaning process was done to remove any residual acrylic adhesive from the strips, that was found to contaminate and weaken the joint. Opening up the $\mathrm{Cu}$-tape cut that exposed the conductor, reduced the tendency of the tape to wick solder from the conductor. The problems of poor quality solder joints diminished significantly after these changes were made and by improving the handling procedures for the final planes, to reduce the chance of flexing.

As of this publication 72 planes were manufactured and 24 have been installed in the detector and are in use during data taking.

\section{Acknowledgments}

The successful design and construction of this detector could not have been accomplished without the help of a large number of skilled people. The authors would also like to thank the Italian and US companies for their tremendous contribution in this project.We acknowledge support from the Department of Energy (US).

\section{References}

[1] B. Aubert et al. (BABAR collab.), Nucl. Instr. and Meth. A479, 1-116(2002).

[2] D. Piccolo et al. (BABAR collab.), Nucl. Instr. and Meth. A477, 435$439(2002)$. 
[3] E. Iarocci, Nucl. Instr. and Meth. A217, 30-42(1983).

[4] M. G. Catanesi et al., Nucl. Instr. and Meth. A247, 438-444(1986).

[5] G. T. J Arnison et al., Nucl. Instr. and Meth. A294, 431-438(1990).

[6] A. C. Benvenuti et al., Nucl. Instr. and Meth. A290, 353-369(1990).

[7] V. Cianciolo et al., Private note.

[8] Gould Electronics Inc, P.O.Box 642441, Pittsburg, PA 15264-2441.

[9] MACTAC, P.O.BOX 945815, Atlanta, GA 30394-5815.

[10] Universal Tape Co, 110 West New Jersey Ave, Sommers Pt, New Jersey 082440-1765.

[11] PARLEX Corp, 1756 Junction Ave, San Jose, CA 95112.

[12] www.amp.com, Part No. 487406-2.

[13] CABLE Connection, 102 Cooper Court, Los Gatos, CA 95043.

[14] Straub Design Company, 2238 Florida Avenue South, Minneapolis, MN 55426. 